\title{
Assessment of the Induced Electric Fields in a Carbon-Fiber Electrical Vehicle Equipped with a Wireless Power Transfer System
}

\author{
Valerio De Santis ${ }^{1}$, Tommaso Campi ${ }^{1, *(1)}$, Silvano Cruciani ${ }^{1}$ (1) , Ilkka Laakso ${ }^{2}$ and \\ Mauro Feliziani ${ }^{1}$ \\ 1 Department of Industrial and Information Engineering and Economics, University of L'Aquila, \\ 67100 L'Aquila, Italy; valerio.desantis@univaq.it (V.D.S.); silvano.cruciani@univaq.it (S.C.); \\ mauro.feliziani@univaq.it (M.F.) \\ 2 Department of Electrical Engineering and Automation, Aalto University, 02150 Espoo, Finland; \\ laakso@aalto.fi \\ * Correspondence: tommaso.campi8888@gmail.com; Tel.: +39-0862-434421
}

Received: 13 February 2018; Accepted: 16 March 2018; Published: 18 March 2018

\begin{abstract}
In this study, the electric field induced inside two realistic anatomical models placed near or inside an electric vehicle made of carbon-fiber composite while charging its battery with a wireless power transfer (WPT) system has been investigated. The WPT source consists of two parallel inductive coils operating with a power output of $7.7 \mathrm{~kW}$ at two different frequencies of 85 and $150 \mathrm{kHz}$. Since a misalignment between the primary and the secondary coil creates higher induced fields, a misalignment of $20 \mathrm{~cm}$ is also considered as the worst-case exposure condition. The analysis of the obtained results shows that the International Commission on Non-Ionizing Radiation Protection (ICNIRP) basic restrictions are exceeded by $1.3 \mathrm{~dB}$ and $4.8 \mathrm{~dB}$ for the aligned and misaligned coil positions, respectively. This exceedance is however confined only in a small area of the driver's foot.
\end{abstract}

Keywords: carbon-fiber composite; electric vehicles (EVs); numerical dosimetry; wireless power transfer (WPT)

\section{Introduction}

Wireless power transfer (WPT) techniques have recently been investigated for various applications, including mobile electronics, home appliances, and electric vehicles (EVs) [1,2]. The latter has received increased attention due to a growing concern for the air pollution produced by conventional diesel/gasoline powered cars. EVs are equipped with large batteries that must be efficiently and rapidly recharged. These batteries can be charged via a WPT technology based on resonant inductive coupling to increase comfort and electrical safety in the charging process [1,2]. WPT can also be used for charging autonomous vehicles.

The growing integration of various electric and electronic devices toward autonomous vehicle technologies has increased the need to deal with electromagnetic compatibility (EMC) and electromagnetic field (EMF) safety issues. Therefore, one of the main concerns in wireless charging of EVs is the compliance of the emitted field levels with the EMF safety standards [3,4].

The International Commission on Non-Ionizing Radiation Protection (ICNIRP) specifies human exposure limits in terms of reference levels (RLs), i.e., measurable fields in air without the presence of the exposed human body, and basic restrictions (BRs), i.e., electromagnetic quantities induced inside the human body to protect people against short-term or acute effects [3]. Compliance assessment of the latter limit is not mandatory if compliance with the former is assured, otherwise it is needed. 
Several authors already performed the evaluation of the induced electric fields produced by EV-WPT systems [5-10]. However, in these studies, considerable simplified assumptions (e.g., body postures or perfect electric conductive conditions for the car body) were made and therefore the dosimetric evaluation was altered by these factors. Moreover, in this study, a dosimetric analysis considering a car bodyshell in carbon fiber (CF) composite is performed for the first time. This topic is very important considering that in the future, to reduce the weight and the emission of the vehicles, advanced lightweight materials—such as CF composite—will be widely used [11].

In recent studies from the authors [12,13], magnetic field levels emitted by a WPT system were evaluated for three different material compositions of the car platform and chassis: steel, aluminum, and CF composite. The analysis of the obtained results showed that the ICNIRP RLs were exceeded inside the car only for a CF composite chassis. In this study, a dosimetric analysis is therefore performed for this configuration to compare the induced electric fields inside realistic anatomical models with the ICNIRP BRs, as requested by the EMF safety standards. The condition with misaligned WPT parallel coils is also investigated to consider the worst-case exposure scenario.

\section{Models and Methods}

\subsection{WPT System Configuration}

At the present time, several standards for WPT applications in EVs have been established. Among these, the International Electrotechnical Commission (IEC), with the standard 61980 [14], and the Society of Automotive Engineers (SAE), with the standard J2954 [14], are the most adopted. They mainly specify the transfer power and the operational frequency of the WPT system, together with other parameters and specifications. Nowadays, for a medium size car, the maximum transfer power is set to $7.7 \mathrm{~kW}$, while the operational frequency is set to $85 \mathrm{kHz}[14,15]$.

However, an increase of the operational frequency is being considered in future standard development to improve system performance [15]. Thus, two operational frequencies—of $f=85 \mathrm{kHz}$ and $f=150 \mathrm{kHz}$ - have been considered in this study; the first for the abovementioned reasons, while the second because is the maximum admissible frequency for current WPT automotive standards. Frequency increase is also limited by the rise in the complexity of the power electronic components and by the increase of the EMF safety and EMC issues.

The magnetic field is assumed to be generated by the currents flowing into two coaxial planar coils. The electro-geometrical details of the WPT system are provided in $[12,13]$ and therefore are omitted here. For the sake of clarity, Figure 1 shows the WPT coil configuration, while Table 1 summarizes the geometrical parameters. The circuital parameters are instead optimized to efficiently transfer to the load a power $P_{L}=7.7 \mathrm{~kW}$ with the output voltage $V_{\text {out }}=200 \mathrm{~V}$ [16]. For simplicity, the load is modeled as a simple power resistor $R_{L}$ while the source is an ideal sinusoidal voltage generator $V_{G}$ with a series resistance $R_{G}[17,18]$. To improve system performance and reduce magnetic field leakage, some ferrite is used. In particular, six ferrite bars are placed under the primary coil while a ferrite disc shield is placed over the secondary coil, acting as a magnetic shield.

Table 1. Geometry parameters of the considered WPT system as shown in Figure 1.

\begin{tabular}{ccccccccc}
\hline $\begin{array}{c}\mathbf{D}_{\text {coil }} \\
(\mathbf{c m})\end{array}$ & $\begin{array}{c}\mathbf{D}_{\mathbf{1}, \text { OUT }} \\
(\mathbf{c m})\end{array}$ & $\begin{array}{c}\mathbf{D}_{\mathbf{2}, \text { OUT }} \\
(\mathbf{c m})\end{array}$ & $\mathbf{D}_{\mathbf{m}}(\mathbf{c m})$ & $\mathbf{l}_{\mathbf{b}}(\mathbf{c m})$ & $\mathbf{w}_{\mathbf{b}}(\mathbf{c m})$ & $\mathbf{h}_{\mathbf{b}}(\mathbf{c m})$ & $\mathbf{D}_{\mathbf{s h}}(\mathbf{c m})$ & $\mathbf{H}_{\mathbf{s h}}(\mathbf{c m})$ \\
\hline 20 & 50 & 40 & 0.8 & 9.3 & 5.8 & 0.4 & 0.5 & 0.2 \\
\hline
\end{tabular}




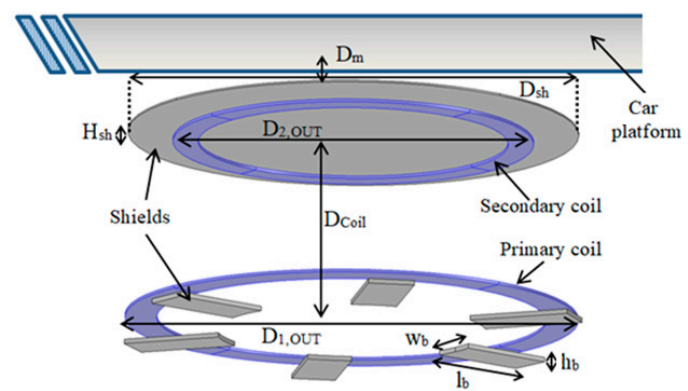

(a)

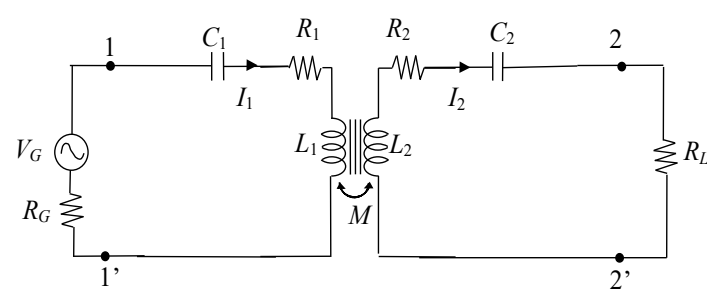

(b)

Figure 1. WPT coil configuration (primary coil on the road and secondary coil below the car platform) (a). WPT equivalent circuit (b).

\subsection{EV, Human Body Models, and Exposure Scenario}

To evaluate the electric fields induced in a human being close to or inside an EV when charging the battery, a 3-D model of the car body has been created (see Figure 2). The car outer dimensions are: $l_{x}=4.3 \mathrm{~m}, l_{y}=1.7 \mathrm{~m}$ and $l_{z}=1.2 \mathrm{~m}$ (without wheels). The distance between the front and rear wheel axes is $2.6 \mathrm{~m}$, while the distance of the car platform from the ground (when the wheels are in place) is set to $20 \mathrm{~cm}$. The car body is modeled by three different materials (steel, aluminum, and CF), whose thicknesses $(d)$ and electrical conductivities $(\sigma)$ are [12]:

(1) CF composite panels $(d=2 \mathrm{~mm}, \sigma=100 \mathrm{~S} / \mathrm{m})$;

(2) steel panels $\left(d=1.2 \mathrm{~mm}, \sigma=10^{7} \mathrm{~S} / \mathrm{m}\right)$;

(3) aluminum panels $\left(d=2 \mathrm{~mm}, \sigma=3 \times 10^{7} \mathrm{~S} / \mathrm{m}\right)$.

It should be noted that the CF composite panels are assumed to be composed by a homogeneous and isotropic material [13].

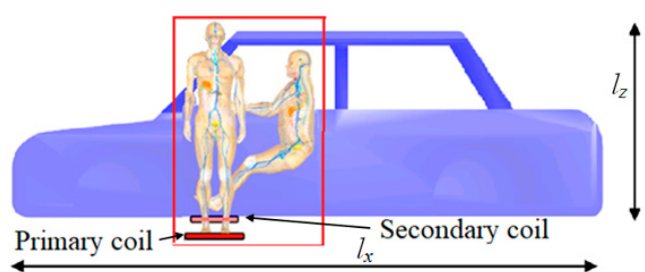

(a)

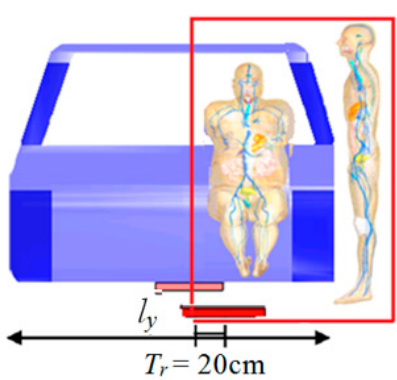

(b)

Figure 2. Configuration of Duke and Fats placed near and inside an EV chassis. (a) Aligned and (b) misaligned coils. The red box is the computational domain for dosimetry assessment.

Two posable adult male human models obtained from the IT'IS Foundation Virtual Population (i.e., 'Duke' and 'Fats') [19] are placed inside the car (driving position) or just in front of the front door (standing position). The choice of these two models is due to their large size, representing a worst-case exposure scenario according to the Faraday law of induction. Dielectric properties of the human tissues have been assigned from the IT'IS database [20], except for the skin where a higher conductivity value has been adopted [21,22].

Since a misalignment between the primary and the secondary coil creates higher induced fields in the human body due to the more leakage of the magnetic field and to the higher current on the coils, a coil misalignment $T_{r}=20 \mathrm{~cm}$ is considered as worst-case exposure condition. Figure 2 shows the CAD model of the car together with the posed human models for the aligned and misaligned coil 
positions. The distance between the driver heel and the secondary coil is $5 \mathrm{~cm}$, accounting for shoes, car floor, and ferrite shield.

\subsection{External Magnetic Field Calculation}

The external magnetic field is evaluated using the COMSOL Multiphysics software. A CAD model of the coils of the WPT system has also been realized and placed under the body floor of the car. First, the lumped parameters of the coils are extracted for each case applying Ohm's law [18]. Then, these parameters are used to analyze the WPT equivalent circuit. Finally, the values of the current on the coils obtained by the circuital analysis are assigned in COMSOL to calculate the magnetic field behavior. The resulting magnetic field is then exported with a grid resolution of $1 \mathrm{~mm}$.

\subsection{Internal Electric Field Calculation}

The induced electric field is evaluated using the Sim4Life platform [23]. Specifically, the low-frequency (LF) magneto-quasi-static (MQS) solver has been used, as it has shown to be working up to about $10 \mathrm{MHz}[24,25]$. The LF-MQS solver is based on the scalar potential finite element (SPFE) method. In this method, the electric field is obtained starting from the knowledge of the magnetic vector potential A. The latter is provided in Sim4Life as an 'external' source term from the previously obtained results. The magnetic flux density (B) and vector potential (A) are indeed exported in COMSOL for the several EV-WPT scenarios (aligned and misaligned coils) without the presence of the human body models. This is because the magnetic field is not altered by biological tissues at these frequencies [23-25]. A fixed grid resolution of $1 \mathrm{~mm}$ is used to discretize the computational domain comprised of the two anatomical models (see Figure 2). The electric field is then averaged over a $2 \times 2 \times 2 \mathrm{~mm}^{3}$ cube according to the ICNIRP guidelines [3]. The 99th percentile is instead not used after considerations from several research groups [26-30].

\section{Results}

\subsection{WPT Circuit Model}

The calculation of the electrical quantities of the system, in particular of the current flowing in the coils, is of paramount importance to calculate the magnetic field. For the aligned coil condition, some results have already been shown in [12]. However, in the case of a misalignment between the coils, the coupling factor between them is drastically reduced [17]. This condition leads to an important increase of the current in the coils respect to the case where the coils are perfectly aligned. Thus, the electrical quantities of the system considering the three chassis materials and the two operational frequencies of 85 and $150 \mathrm{kHz}$ are reported in Tables 2 and 3, respectively. As can be observed, the current on the primary coil is much higher at the frequency of $85 \mathrm{kHz}$ than that at $150 \mathrm{kHz}$ due to the higher efficiency obtained by the increase of the operational frequency [12]. This leads to a reduction of the magnetic field at the higher frequencies. It should be noted that the current on the secondary coil is equal for all considered cases since the load $R_{L}$ is constant and the output power $P_{L}$ is kept fixed while adjusting the input voltage [16]. In the following, the magnetic field distribution is reported only for the worst-case at the frequency of $85 \mathrm{kHz}$.

Table 2. WPT lumped inductances and r.m.s. currents at $85 \mathrm{kHz}$.

\begin{tabular}{ccccccc}
\hline EV Bodyshell & $\boldsymbol{L}_{\mathbf{1}}(\boldsymbol{\mu H})$ & $\boldsymbol{L}_{\mathbf{2}}(\boldsymbol{\mu H})$ & $\boldsymbol{M}(\boldsymbol{\mu H})$ & $\boldsymbol{k}$ & $\boldsymbol{I}_{\mathbf{1}}(\mathrm{A})$ & $\boldsymbol{I}_{\mathbf{2}}(\mathrm{A})$ \\
\hline Carbon fiber & 125.2 & 89.4 & 7.4 & 0.069 & 50.3 & 39.2 \\
composite & 125.9 & 88.4 & 7.1 & 0.065 & 55.1 & 39.2 \\
Aluminum & 124.1 & 86.1 & 6.5 & 0.061 & 59.1 & 39.2 \\
Steel &
\end{tabular}


Table 3. WPT lumped inductances and r.m.s. currents at $150 \mathrm{kHz}$.

\begin{tabular}{ccccccc}
\hline EV Chassis & $\boldsymbol{L}_{\mathbf{1}}(\boldsymbol{\mu H})$ & $\boldsymbol{L}_{\mathbf{2}}(\boldsymbol{\mu H})$ & $\boldsymbol{M}(\boldsymbol{\mu H})$ & $\boldsymbol{k}$ & $\boldsymbol{I}_{\mathbf{1}}(\mathbf{A})$ & $\boldsymbol{I}_{\mathbf{2}}(\mathbf{A})$ \\
\hline Carbon fiber & 124.8 & 88.2 & 7.3 & 0.070 & 26.5 & 39.2 \\
composite & 124.5 & 87.3 & 7.1 & 0.068 & 29.7 & 39.2 \\
Aluminum & 123.5 & 85.4 & 6.4 & 0.062 & 32.5 & 39.2 \\
$\quad$ Steel & & &
\end{tabular}

\subsection{External Magnetic Field}

The magnetic field distributions at the frequency of $85 \mathrm{kHz}$ are hereby reported in Figures 3 and 4 for the conductive chassis materials (aluminum and steel) and CF composite, respectively. In the figures, the anatomical models are overlaid to facilitate the comparison with the exposure limits. For the conductive materials (see Figure 3), only the worse misalignment condition is analyzed. As can be observed, the ICNIRP RL (yellow line) is in this case never exceeded inside human bodies. This is because the presence of a conductive chassis effectively shields the magnetic field.

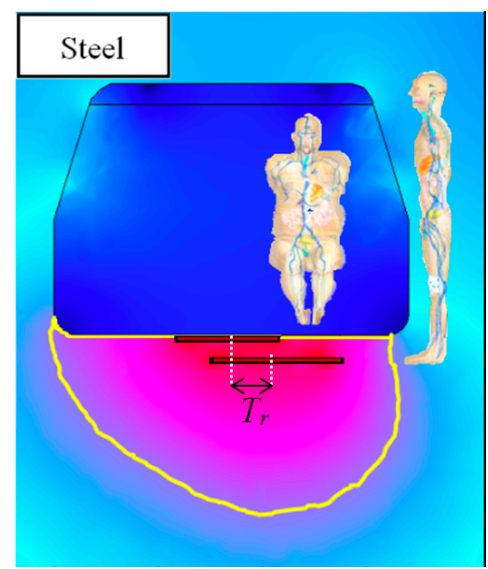

(a)

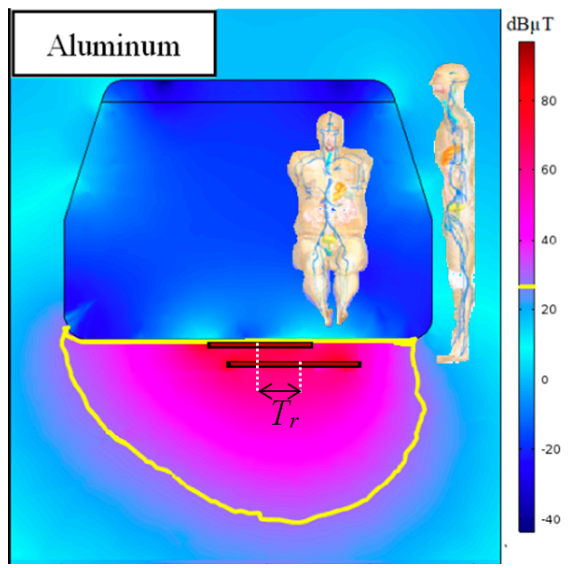

(b)

Figure 3. Magnetic flux density distribution for the misaligned coil position. (a) Steel chassis; (b) aluminum chassis. The yellow line represents the ICNIRP limit $(\mathrm{RL}=27 \mu \mathrm{T}=28.63 \mathrm{~dB} \mu \mathrm{T})$.

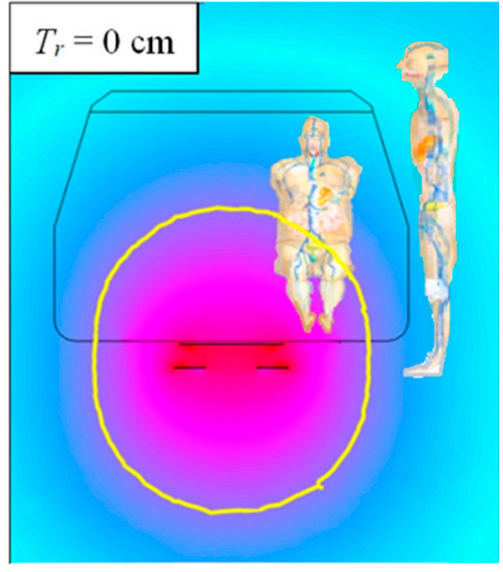

(a)

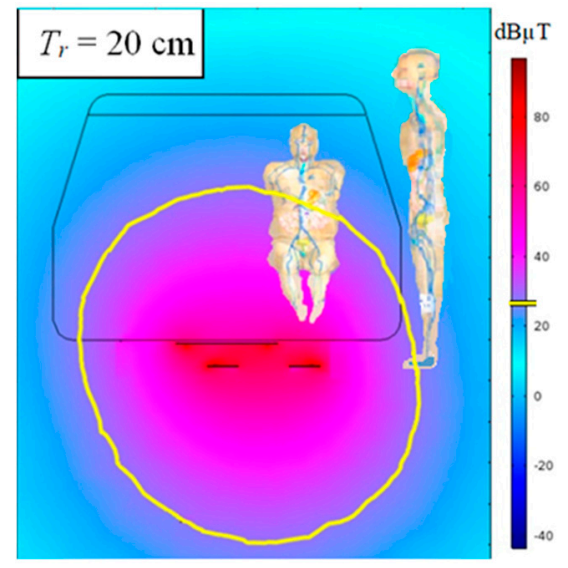

(b)

Figure 4. Magnetic flux density distribution for the aligned (a) and misaligned (b) coil positions in the case of CF composite chassis. The yellow line represents the ICNIRP limit $(R L=27 \mu \mathrm{T}=28.63 \mathrm{~dB} \mu \mathrm{T})$. 
Figure 4 shows the $B$-field distribution for the aligned and misaligned coil positions for the CF composite chassis. As can be observed, the misalignment condition (see Figure $4 \mathrm{~b}$ ) leads to an important increase of the magnetic field level. For this case, the ICNIRP RL is exceeded in both the driver and a standing person outside the car, while for the aligned condition the RL is exceeded only in the driver. This is both due to the increase of the coil currents and the reduction of the distance between the transmitting coil and the person outside the vehicle in the misaligned position.

The maximum magnetic field for both anatomical models are reported in Table 4. As can be observed, the overexposure compared to the RL can reach $16 \mathrm{~dB}$ for the aligned condition and $24.2 \mathrm{~dB}$ for the misaligned condition. The exceedance of the RL for the CF chassis makes the verification of the compliance with the BRs in terms of internal electric fields necessary.

Table 4. Maximum magnetic field for both anatomical models.

\begin{tabular}{ccccc}
\hline EV Chassis & $\boldsymbol{T}_{\boldsymbol{r}}(\mathbf{c m})$ & $\boldsymbol{B}_{\max }(\mathbf{d B} \boldsymbol{\mu} \mathbf{T})$ Driver & $\boldsymbol{B}_{\max }(\mathbf{d B} \boldsymbol{\mu} \mathbf{T})$ Standing & Overexposure $(\mathbf{d B})$ \\
\hline Carbon fiber & 0 & 44.6 & 18.5 & 16.0 \\
Composite & 20 & 52.8 & 32.3 & 24.2 \\
Aluminum & 20 & -4.2 & 19.1 & $/$ \\
Steel & 20 & -18.2 & 20.3 & $/$ \\
\hline
\end{tabular}

\subsection{Induced Electric Field}

The electric field distribution induced inside the two anatomical models is reported in Figures 5 and 6 for the aligned and misaligned coil position, respectively. The obtained results, shown as the ICNIRP BRs, are exceeded only in a few and small portions of the feet for the driving passenger, while no exceedance is observed for a standing person outside the car.
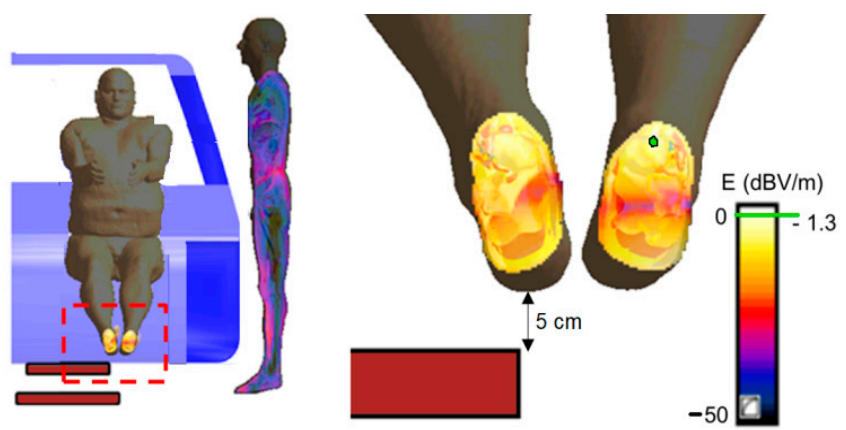

Figure 5. Induced electric field inside Duke and Fats for the aligned coil position. E-field normalized to the peak value of $13.3 \mathrm{~V} / \mathrm{m} . \mathrm{BR}=11.48 \mathrm{~V} / \mathrm{m} \approx-1.3 \mathrm{dBV} / \mathrm{m}$ (green area is the portion where the $\mathrm{BR}$ is exceeded).
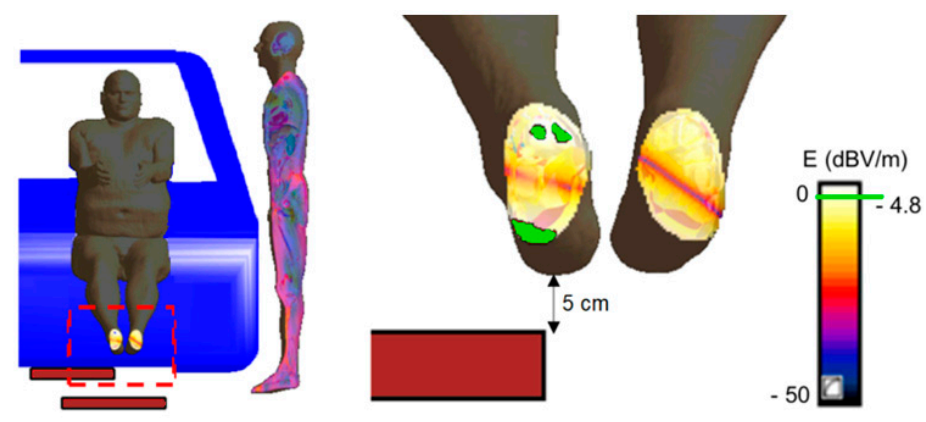

Figure 6. Induced electric field inside Duke and Fats for the misaligned coil position. E-field normalized to the peak value of $19.9 \mathrm{~V} / \mathrm{m} . \mathrm{BR}=11.48 \mathrm{~V} / \mathrm{m} \approx-4.8 \mathrm{dBV} / \mathrm{m}$ (green area is the portion where the BR is exceeded). 
To quantify the obtained results, ICNIRP basic restrictions are exceeded by 1.3 and $4.8 \mathrm{~dB}$ for the aligned and misaligned coil position, respectively. Table 5 shows the peak induced fields for other considered exposure scenarios-e.g., exchanged position between Duke and Fats-and the higher frequency of $150 \mathrm{kHz}$ for the misaligned coils. As expected, no relevant changes in the overexposure is observed due to the frequency scaling and similar anatomical structure of feet and heels.

Table 5. Comparison of the peak induced electric field averaged over a $2 \times 2 \times 2 \mathrm{~mm}^{3}$ cube and the BR for the considered exposure scenarios.

\begin{tabular}{cccccc}
\hline Standing \& Driving & Coil Position & $f(\mathbf{k H z})$ & $\boldsymbol{E}(\mathbf{V} / \mathbf{m})$ & $\boldsymbol{B R} \mathbf{( V / m )}$ & Overexposure $(\mathbf{d B})$ \\
\hline Duke \& Fats & Aligned & 85 & 13.3 & 11.4 & 1.28 \\
Duke \& Fats & Misaligned & 85 & 19.9 & 11.4 & 4.77 \\
Fats \& Duke & Misaligned & 85 & 19.6 & 11.4 & 4.65 \\
Fats \& Duke & Misaligned & 150 & 35 & 20.2 & 4.75 \\
\hline
\end{tabular}

\section{Conclusions}

In this study, both the external magnetic field and internal electric field induced inside two realistic anatomical models placed near or inside a carbon-fiber electric vehicle recharging its battery have been evaluated. From the obtained results, it is shown that the ICNIRP RLs can be exceeded by a factor up to $24.2 \mathrm{~dB}$, while the basic restrictions are exceeded by 1.3 and $4.8 \mathrm{~dB}$ for the aligned and misaligned coil positions, respectively. This exceedance is likely confined in a small area of the driver foot only. However, to drastically reduce the magnetic field inside the vehicle, a further shielding system must be introduced. By adding a thin layer of conductive material between the receiving coil and the car platform, for example, as proposed in [13].

As regards the comparison with previous studies, the induced electric fields were exceeded in [5] by a factor of 7.7 for an aligned WPT coil system, however the inductive power transfer system was considered with a quite unrealistic anatomical posture of a lying person reaching out with his right arm. No exceedance of the basic restrictions were instead observed in [6-11] for different postures and anatomical models. However in those studies, the car body and chassis was considered as a metallic or perfect electric conductor notably reducing the magnetic field penetrating inside the vehicle only from the windows. Here, a carbon-fiber chassis is instead considered as the worst-case scenario, though a thin metallic shield could be employed just above the secondary coil to mitigate the field penetrating the car, as previously stated in $[12,13]$.

It should also be noted that our work is a dosimetric study for a specific application only, assuming the magnetic field generated by two parallel coils in presence of ferrite. Thus, an uncertainty budget (by varying WPT source, dielectric properties, grid resolution, anatomical models, LF solvers, and so on) is not performed as our results are not meant to be generalized to all EV-WPT systems. However, the simulations of the WPT coil system, car body, and human models are realistic and show that limiting human exposure is an important design constraint for WPT systems in EVs made of composite materials.

Acknowledgments: The authors would like to thank the graduate students, G. Valerio and M. Petrongolo-from the University of L'Aquila, L'Aquila, Italy_for developing the CAD model of the car and for posing the anatomical models inside it.

Author Contributions: Tommaso Campi, Silvano Cruciani, and Mauro Feliziani designed the WPT system and carried out the simulations for magnetic field assessment in EV. Valerio De Santis and Ilkka Laakso carried out the dosimetric simulations and post processing analysis. All authors provided critical feedback, improved the simulations accuracy, analyzed data and wrote the paper.

Conflicts of Interest: The authors declare no conflict of interest. The founding sponsors had no role in the design of the study; in the collection, analyses, or interpretation of data; in the writing of the manuscript, and in the decision to publish the results. 
Founding: Valerio De Santis was supported by the Italian Ministry of Education, Universities and Research (MIUR), grant no. PGR13CK2WU. Ilkka Laakso was supported by the Academy of Finland, grant no. 285610.

\section{References}

1. Covic, G.A.; Boys, J.T. Inductive power transfer. Proc. IEEE 2013, 101, 1276-1289. [CrossRef]

2. Shinohara, N. Power without wires. IEEE Microw. Mag. 2011, 11, 64-73. [CrossRef]

3. International Commission on Non-Ionizing Radiation Protection. Guidelines for limiting exposure to time-varying electric and magnetic fields for low frequencies (1 Hz-100 kHz). Health Phys. 2010, 99, 818-836.

4. IEEE Std. C95.1-2005, IEEE Standard for Safety Levels with Respect to Human Exposure to Radio Frequency Electromagnetic Fields, 3 kHz to 300 GHz; IEEE: New York, NY, USA, 2006; pp. 1-238.

5. Cimala, C.; Clemens, M.; Streckert, J.; Schmuelling, B. High-resolution magnetic-field exposure simulations of automotive inductive power-transfer systems using a scaled-frequency finite difference time domain approach with multi-GPU acceleration. Int. J. Numer. Model 2017, e2231, 1-5. [CrossRef]

6. Sunohara, T.; Hirata, A.; Laakso, I.; Onishi, T. Analysis of in situ electric field and specific absorption rate in human models for wireless power transfer system with induction coupling. Phys. Med. Biol. 2014, 59, 3721-3735. [CrossRef] [PubMed]

7. Shimamoto, T.; Laakso, I.; Hirata, A. In-situ electric field in human body model in different postures for wireless power transfer system in an electrical vehicle. Phys. Med. Biol. 2015, 60, 163-173. [CrossRef] [PubMed]

8. Chakarothai, J.; Wake, K.; Arima, T.; Watanabe, S.; Uno, T. Exposure evaluation of an actual Wireless Power Transfer system for an electric vehicle with near-field measurement. IEEE Trans. Microw. Theory Tech. 2018, 66, 1543-1552. [CrossRef]

9. Park, S. Evaluation of Electromagnetic Exposure during $85 \mathrm{kHz}$ Wireless Power Transfer for Electric Vehicles. IEEE Trans. Magn. 2018, 54, 1-8. [CrossRef]

10. Pinto, R.; Lopresto, V.; Genovese, A. Human exposure to wireless power transfer systems: A numerical dosimetric study. In Proceedings of the 11th European Conference on Antennas and Propagation (EUCAP), Paris, France, 19-24 March 2017.

11. Romo, J.; Cañibano, E.; Merino, J.C. Lightweighting and passive safety for urban electric vehicle. In Proceedings of the Electric Vehicles International Conference (EV2017), Bucharest, Romania, 5-6 October 2017.

12. Campi, T.; Cruciani, S.; De Santis, V.; Maradei, F.; Feliziani, M. Numerical characterization of the magnetic field in electrical vehicles equipped with a WPT system. Wirel. Power Transf. 2017, 4, 78-87. [CrossRef]

13. Campi, T.; Cruciani, S.; De Santis, V.; Maradei, F.; Feliziani, M. Magnetic field behavior in a carbon-fiber Electrical Vehicle charged by a Wireless Power Transfer system. In Proceedings of the 2017 IEEE International Symposium on Electromagnetic Compatibility (EMC), Angers, France, 4-8 September 2017.

14. IEC 61980-1. Electric Vehicle Wireless Power Transfer (WPT) Systems_Part 1: General Requirements; International Electrotechnical Commission: Geneva Switzerland, 2015.

15. SAE TIR J2954. Wireless Power Transfer for Light-Duty Plug-In/Electric Vehicles and Alignment Methodology; SAE International: Warrendale, PA, USA, 2016.

16. Campi, T.; Cruciani, S.; De Santis, V.; Feliziani, M. EMF safety and thermal aspects in a pacemaker equipped with a Wireless Power Transfer system working at low frequency. IEEE Trans. Microw. Theory Tech. 2016, 64, 375-382. [CrossRef]

17. Campi, T.; Cruciani, S.; Maradei, F.; Feliziani, M. Near field reduction in a Wireless Power Transfer System using LCC compensation. IEEE Trans. Electromagn. Compat. 2017, 59, 686-694. [CrossRef]

18. Cruciani, S.; Campi, T.; Feliziani, M.; Maradei, F. Optimum coil configuration of wireless power transfer system in presence of shields. In Proceedings of the 2015 IEEE International Symposium on Electromagnetic Compatibility (EMC), Dresden, Germany, 16-22 August 2015; pp. 720-725.

19. Gosselin, M.C.; Neufeld, E.; Moser, H.; Huber, E.; Farcito, S.; Gerber, L.; Jedensjö, M.; Hilber, I.; Di Gennaro, F.; Lloyd, B.; et al. Development of a new generation of high-resolution anatomical models for medical device evaluation: The Virtual Population 3.0. Phys. Med. Biol. 2014, 59, 5287-5303. [CrossRef] [PubMed] 
20. Hasgall, P.A.; Di Gennaro, F.; Baumgartner, C.; Neufeld, E.; Gosselin, M.C.; Payne, D.; Klingenböck, A.; Kuster, N. IT'IS Database for Thermal and Electromagnetic Parameters of Biological Tissues; Version 3.0; ScienceOpen, Inc.: Boston, MA, USA, 2015.

21. De Santis, V.; Chen, X.L.; Laakso, I.; Hirata, A. An equivalent skin conductivity for LF magnetic field dosimetry. Biomed. Phys. Eng. Express 2015, 1, 1-10. [CrossRef]

22. De Santis, V.; Douglas, M.G.; Chen, X.L.; Kuster, N. Impact of the skin conductivity and displacement currents on LF dosimetry. In Proceedings of the 2012 IEEE International Symposium on Electromagnetic Compatibility (EMC), Rome, Italy, 4-8 September 2012.

23. Sim4Life-v3.2. SIMulation 4 LIFE Science. Available online: www.zurichmedtech.com/sim4life (accessed on 15 October 2017).

24. Hand, J.W. Modelling the interaction of electromagnetic fields (10 MHz-10 GHz) with the human body: Methods and applications. Phys. Med. Biol. 2008, 53, R243-R286. [CrossRef] [PubMed]

25. Hirata, A.; Ito, F.; Laakso, I. Confirmation of quasi-static approximation in SAR evaluation for a wireless power transfer system. Phys. Med. Biol. 2013, 58, N241-N249. [CrossRef] [PubMed]

26. Chen, X.L.; Benkler, S.; Chavannes, N.; De Santis, V.; Bakker, J.; van Rhoon, G.; Mosig, J.; Kuster, N. Analysis of human brain exposure to low-frequency magnetic fields: A numerical assessment of spatially averaged electric fields and exposure limits. Bioelectromagnetics 2013, 34, 375-384. [CrossRef] [PubMed]

27. De Santis, V.; Chen, X.L. On the issues related to compliance assessment of ICNIRP 2010 basic restrictions. J. Radiat. Prot. 2014, 34, N31-N39. [CrossRef] [PubMed]

28. De Santis, V.; Chen, X.L.; Cruciani, S.; Campi, T.; Feliziani, M. A novel homogenization procedure to model the skin layers in LF numerical dosimetry. Phys. Med. Biol. 2016, 61, R4402-R4411. [CrossRef] [PubMed]

29. Schmid, G.; Cecil, S.; Überbacher, R. On the importance of body posture and skin modelling with respect to in situ electric field strengths in magnetic field exposure scenarios. Phys. Med. Biol. 2016, 61, 4412-4437. [CrossRef] [PubMed]

30. Reilly, J.P.; Hirata, A. Low-frequency electrical dosimetry: Research agenda of the IEEE International Committee on Electromagnetic Safety. Phys. Med. Biol. 2016, 61, R138-R149. [CrossRef] [PubMed]

(C) 2018 by the authors. Licensee MDPI, Basel, Switzerland. This article is an open access article distributed under the terms and conditions of the Creative Commons Attribution (CC BY) license (http:/ / creativecommons.org/licenses/by/4.0/). 